\title{
Effect of screening for type 2 diabetes on healthcare costs: a register-based study among 139,075 individuals diagnosed with diabetes in Denmark between 2001 and 2009
}

\author{
Camilla Sorts $\emptyset^{1}$ - Anastasija Komkova ${ }^{1}$ - Annelli Sandbæk ${ }^{2}$ - Simon J. Griffin ${ }^{2,3} \cdot$ Martha Emneus $^{1} \cdot$ Torsten Lauritzen $^{2}$ • \\ Rebecca K. Simmons ${ }^{2,4,5,6}$
}

Received: 24 September 2017 / Accepted: 5 February 2018 / Published online: 16 March 2018

(C) The Author(s) 2018

\begin{abstract}
Aims/hypothesis Trials have not demonstrated benefits to the population of screening for type 2 diabetes. However, there may be cost savings for those found to have diabetes. We therefore aimed to compare healthcare costs among individuals with incident type 2 diabetes in a screened group with those in an unscreened group.

Methods In this register-based, non-randomised controlled trial, eligible individuals were men and women aged 40-69 years without known diabetes who were registered with a general practice in Denmark $(n=1,912,392)$. Between 2001 and 2006, 153,107 individuals registered with 181 practices participating in the Anglo-Danish-Dutch Study of Intensive Treatment in People with Screen Detected Diabetes in Primary Care (ADDITION)-Denmark study were sent a diabetes risk-score questionnaire. Individuals with a moderate-to-high risk were invited to visit their family doctor for assessment of diabetes status and cardiovascular risk (screening group). The 1,759,285 individuals registered with all other practices in Denmark constituted the retrospectively constructed no-screening (control) group. In this post hoc analysis, we identified individuals from the screening and no-screening groups who were diagnosed with diabetes between 2001 and $2009(n=139,075)$. Using national registry data, we quantified the cost of healthcare services in these two groups between 2001 and 2012. From a healthcare sector perspective, we estimated the potential healthcare cost savings for individuals with diabetes that were attributable to the screening programme. Results In the screening group, 27,177 of 153,107 individuals (18\% of those sent a risk-score questionnaire) attended for screening, 1533 of whom were diagnosed with diabetes. Between 2001 and 2009, 13,992 people were newly diagnosed with diabetes in the screening group (including those diagnosed by screening) and 125,083 in the no-screening group. Healthcare costs were significantly lower in the screening group compared with the no-screening group (difference in mean total annual healthcare costs $-€ 889$ per individual with incident diabetes; $95 \% \mathrm{CI}-€ 1196, € 581)$. The screening programme was associated with a cost saving per person with incident diabetes over a 5-year period of $€ 2688$ (95\% CI €1421, €3995).

Conclusions/interpretation Healthcare costs were lower among individuals with incident type 2 diabetes in the screened group compared with the unscreened group. The relatively modest cost of screening per person discovered to have developed diabetes was offset within 2 years by savings in the healthcare system.
\end{abstract}

Electronic supplementary material The online version of this article (https://doi.org/10.1007/s00125-018-4594-2) contains peer-reviewed but unedited supplementary material, which is available to authorised users.

Camilla Sortsø

camillasortsoe@gmail.com

Rebecca K. Simmons

rks34@cam.ac.uk

1 Applied Economics and Health Research Aps, Ewaldsgade 3, 2200 Copenhagen N, Denmark

2 Department of Public Health, Section of General Practice, Aarhus, Denmark
3 Primary Care Unit, Institute of Public Health, Cambridge, UK

4 Danish Diabetes Academy, Odense University Hospital, Odense, Denmark

5 Aarhus Institute of Advanced Studies, Aarhus, Denmark

6 The Healthcare Improvement Studies (THIS) Institute, University of Cambridge School of Clinical Medicine, Cambridge Biomedical Campus, Cambridge CB2 0AH, UK 


\section{Research in context}

\section{What is already known about this subject?}

- Trials have not demonstrated benefits to the population of screening for type 2 diabetes

- For individuals diagnosed with diabetes, however, screening is associated with a reduction in mortality and risk of cardiovascular disease. The potential effect on healthcare costs in this group is unknown

\section{What is the key question?}

- What is the effect of screening on healthcare costs among individuals diagnosed with diabetes in Denmark?

What are the new findings?

- Healthcare costs were lower among individuals with incident type 2 diabetes in a screened group compared with an unscreened group

How might this impact on clinical practice in the foreseeable future?

- Our findings add to the evidence base on the potential cost savings of early detection and treatment among individuals at high risk of diabetes

Keywords ADDITION study $\cdot$ Diabetes $\cdot$ Healthcare costs $\cdot$ Screening

\begin{tabular}{|c|c|}
\hline \multicolumn{2}{|c|}{ Abbreviations } \\
\hline ADDITION & Anglo-Danish-Dutch Study of Intensive \\
\hline & Treatment in People with Screen Detected \\
\hline & Diabetes in Primary Care \\
\hline CVD & Cardiovascular disease \\
\hline GP & General practitioner \\
\hline IHD & Ischaemic heart disease \\
\hline VAT & Value-added tax \\
\hline
\end{tabular}

\section{Introduction}

Although modelling studies indicate that screening for type 2 diabetes might be effective and cost-effective [1-3], trials of population-based screening for type 2 diabetes [4] and related cardiovascular risk factors [5] have not demonstrated beneficial effects at the population level. However, there appear to be benefits for those found to have diabetes. We have previously shown that screening for type 2 diabetes and cardiovascular risk factors does not reduce mortality and cardiovascular disease (CVD) in the general population in Denmark [5], but for individuals diagnosed with diabetes, screening was associated with a reduction in mortality and risk of CVD [6]. Using data from Danish national registers and a retrospectively constructed control group, we extended this analysis to compare total healthcare costs for individuals with incident diabetes in the screening and no-screening groups, and estimated the potential cost savings of the Anglo-Danish-Dutch Study of Intensive Treatment in People with Screen Detected
Diabetes in Primary Care (ADDITION) intervention from a healthcare sector perspective.

\section{Methods}

ADDITION-Europe is a cluster-randomised trial comparing the effects of screening for type 2 diabetes followed by intensive treatment or routine care $[7,8]$. We report here results from a post hoc analysis using data from the screening phase of the Danish arm of the study in conjunction with data from Danish national registers. Ethical approval for the ADDITIONDenmark study was granted by a local scientific committee (number 20000183). As this was a registry-based study using anonymised data, participants did not give informed consent. This approach was approved by the Danish Data Protection Agency and the Danish Health and Medicine Authority.

Screening programme Full details of the programme have been reported elsewhere [5, 6, 9]. In brief, we performed a population-based, stepwise screening programme in people aged 40-69 years without known diabetes between 2001 and $2006[7,8,10]$. All general practices in five out of 16 counties in Denmark (Copenhagen, Aarhus, Ringkoebing, Ribe and South Jutland; $n=744$ ) were invited to take part in ADDITION-Denmark; of these, 209 (28\%) accepted.

Eligible individuals registered with the 181 practices that agreed to take part were sent a diabetes risk-score questionnaire $[8,10]$, with an invitation to visit their general practitioner (GP) for a diabetes test and a cardiovascular risk assessment 
if they scored $\geq 5$ (maximum 15 points), or were invited when visiting the practice for another reason ( $n=35$ practices). No reminders were sent. Participants who attended a screening appointment underwent measurement of height, weight and blood pressure. A capillary blood sample was taken for testing of random blood glucose. A venous blood sample was taken for measurement of total cholesterol and $\mathrm{HbA}_{1 \mathrm{c}}$. GPs were encouraged to calculate participants' scores from European Heart (Systematic COronary Risk Evaluation [SCORE]) [11] charts during the appointment, inform participants about their score and provide appropriate advice and treatment to those at high risk. Individuals with a random blood glucose $\geq 5.5 \mathrm{mmol} / 1$ or $\mathrm{HbA}_{1 \mathrm{c}} \geq 39.9 \mathrm{mmol} / \mathrm{mol}(5.8 \%)$ were invited to return to the practice for a fasting blood glucose capillary test. An OGTT was performed at the same consultation if the fasting blood glucose was $5.6-6.1 \mathrm{mmol} / 1$ and/or the $\mathrm{HbA}_{1 \mathrm{c}} \geq$ $39.9 \mathrm{mmol} / \mathrm{mol}(5.8 \%)$. WHO 1999 criteria were used to diagnose diabetes [12].

In the intervention group, participants diagnosed with type 2 diabetes were subsequently managed according to the treatment regimens to which their practice was allocated: routine care or intensive treatment [9]. Intensive treatment included: small group or practice-based educational meetings with GPs and nurses to discuss treatment targets, algorithms and lifestyle advice; audit and feedback in group meetings up to twice per year or coordinated by post; and patient education materials. GPs underwent a 1.5 day educational seminar to train in motivational interviewing to encourage lifestyle change.

Sampling frame We identified all eligible individuals in the original ADDITION-Denmark study $(n=153,107)$, including those who did not attend for screening, in the Danish National Diabetes Register [13] (the screening group). Using the same registry, we also identified all individuals aged 40-69 years without known diabetes who, between 2001 and 2006, were registered with general practices that were not invited to take part in ADDITION-Denmark or who declined to take part in ADDITION-Denmark $(n=1,759,285)$ (the no-screening control group). We then identified individuals from the screening and no-screening groups who were diagnosed with incident diabetes between 1 January 2001 and 31 December 2009 (Fig. 1). We included individuals diagnosed with diabetes during this period based on recent estimates of lead time, which suggest that around 2.2 years elapses between detection by screening and clinical diagnosis [6]. Including individuals diagnosed with diabetes in the 3 years (2006-2009) after the end of the ADDITION screening phase (2001-2006) would therefore capture most individuals in the no-screening group who could have been diagnosed by screening if they had been in the screening group. Our definition of incident diabetes for both groups was a proxy measure based on date of inclusion in the Danish National Diabetes Register [14].

Data We linked information about individuals diagnosed with diabetes to other Danish registers using unique civil registration numbers. We retrieved information on age, sex, education, immigration/emigration, citizenship, redeemed cardioprotective medication and chronic disease. Education was categorised according to UNESCO's International Standard Classification of Education [15]. We grouped data on citizenship into European and non-European as a proxy for ethnicity. Data on healthcare service usage in primary and secondary care, as well as information on redeemed medication, were retrieved from the National Patient Register, the National Health Insurance Register and the National Prescription Register [16].
Fig. 1 Visual representation of the sampling frame. The ' $\mathrm{S}$ ' in a blue circle denotes individuals detected by the ADDITION stepwise screening programme. The ' $C$ ' in a red circle denotes individuals with clinically diagnosed diabetes. Reproduced from Simmons et al. [6] under the terms of the CC BY 4.0 Attribution License (http:// creativecommons.org/licenses/ by/4.0/)

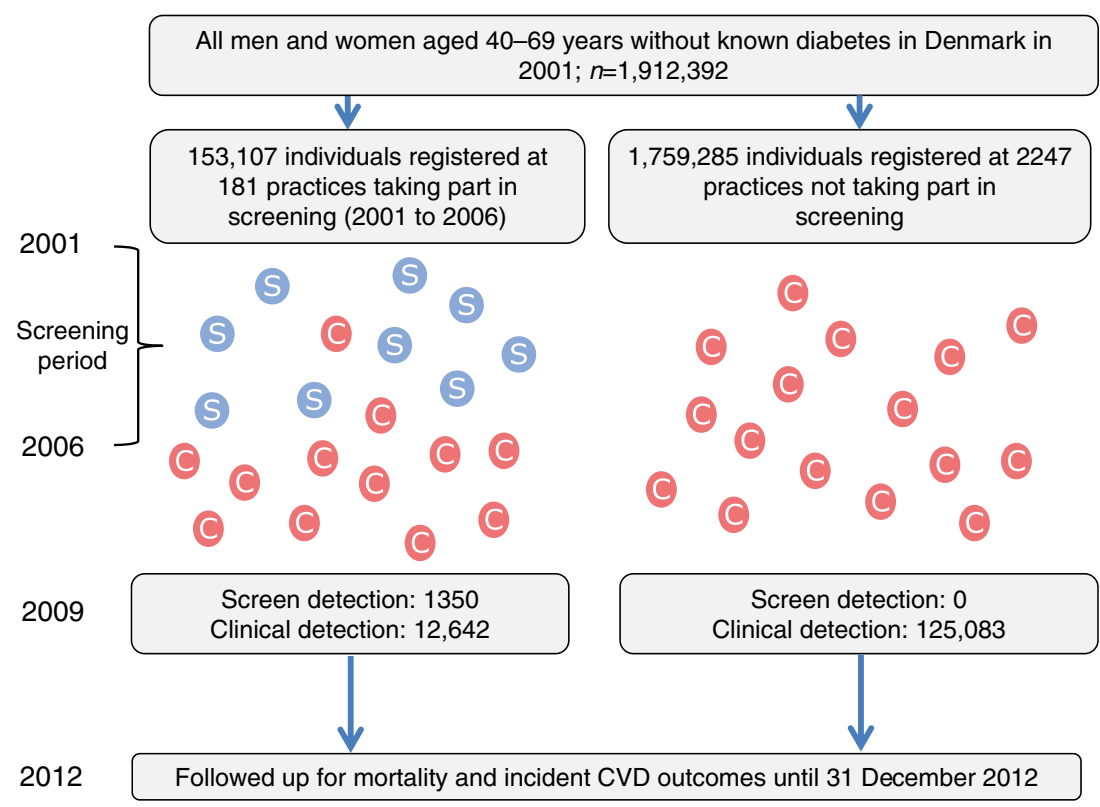




\section{Costs}

The costs of ADDITION-Denmark include the cost of the screening programme and the cost of the intensive treatment programme (for those found with screen-detected diabetes who were randomised to this arm). Healthcare costs include total costs of healthcare service usage and redeemed medication for all individuals in our sampling frame during the follow-up period. All costs were calculated in Danish krone (DKK) and converted to Euros (conversion rate $€ 1=7.44$ DKK), and were from a healthcare sector perspective; for example, all costs, included overhead costs and participants' own payment for redeemed medication.

The cost of the screening programme has previously been reported [17]. The average cost of detecting an individual with previously undiagnosed type 2 diabetes was estimated by Dalsgaard and colleagues in 2010 to be $€ 936$ [17], including the cost of sending the invitation letter, and the cost of the screening consultations and laboratory tests. We multiplied GP fees by 1.438 to capture overhead costs, as Danish GPs are paid on a combined capitation and fee-for-service scheme.

The cost of the intensive treatment intervention includes: the cost of small group or practice-based educational meetings with GPs and nurses to discuss treatment targets, algorithms and lifestyle advice; audit and feedback in group meetings up to twice per year or coordinated by post; patient education materials; and small financial incentives for GPs [9]. In addition, 64 GPs participated in a 1.5 day educational seminar on motivational interviewing. Summing the costs of the screening programme and the intensive treatment costs provided an estimate of $€ 967$ per individual with incident diabetes.

The cost of healthcare usage was defined as opportunity costs using market values (excluding value-added tax [VAT]) $[18,19]$. We quantified the healthcare costs in the screening and no-screening groups using individual-level register data. These data allowed us to estimate the cost of: (1) inpatient healthcare utilisation (data available for 2007-2012); (2) outpatient healthcare utilisation (data available for 2007-2012); (3) primary care utilisation (data available for 2001-2012); and (4) redeemed medications (data available for 20012012). Table 1 describes the different cost units and our methods for calculating them.

Statistical analysis We summarised characteristics of all individuals diagnosed with incident diabetes between 2001 and 2009 separately in the screening and no-screening groups. Date of entry to the study was the date of inclusion in the diabetes register. All analyses were completed using Stata version 14.1 (Stata, College Station, TX, USA). Statistical significance was inferred at a two-tailed $p<0.05$. We calculated robust standard errors to allow for clustering of participants within practices.
Cost analysis We quantified healthcare usage and calculated average healthcare costs in each group over the follow-up period by dividing the total healthcare costs for each individual by the time they spent in the study. As data from the health registers were available for different time periods, we conducted analyses for two time periods: 2001-2012 (with an average of 7.0 years follow-up; $n=139,048$ ) and 2007-2012 (with an average of 5.2 years follow-up; $n=133,307)$. In this second analysis, we only included individuals who were alive and living in Denmark on 1 January 2007.

To examine potential differences in healthcare costs between the groups, we used ordinary least squares regression, adjusting for age, sex, education and GP county. We also controlled for prevalent chronic disease at diagnosis (ischaemic heart disease [IHD], stroke and cancer). We quantified costs from more than 3 years before diagnosis to more than 6 years after diagnosis using difference-in-difference regression on panel data (data covering more years over a time period) with mean correction for confounders. The difference-in-difference method allowed us to estimate the 'isolated' effect of the ADDITION intervention on healthcare costs by examining the differences between the screening group and the no-screening group within the periods before and after diagnosis. We included mean-adjusted covariates in the regression, for which we applied effect coding for the categorical variables [20] in order to adjust for the demographic, epidemiological and regional differences. We were therefore able to interpret the constant term as the predicted healthcare costs for an individual in the no-screening group in the year of diagnosis.

To estimate the potential economic benefits of the screening programme among individuals with incident diabetes, we subtracted the cost of the screening programme from the estimated healthcare cost savings accruing in the screening group. We calculated cost savings over a 5 year period after the screening phase using an annual discount rate of 5\%.

Sensitivity analysis We also examined the difference in healthcare costs between the screening group assigned to routine care only and the no-screening group.

\section{Results}

Population characteristics of 153,107 eligible people in the screening group who were sent a diabetes risk-score questionnaire, 27,177 (18\%) attended their GP for a diabetes test and a cardiovascular risk assessment. A total of 1533 participants ( $1 \%$ of those eligible for screening) were diagnosed with diabetes; 1406 of these were subsequently included in the diabetes register. There were $1,759,285$ individuals in the no-screening group. 
Table 1 Cost units and methods of calculation for cost components

\begin{tabular}{|c|c|}
\hline Cost component & Cost unit and method of calculation \\
\hline $\begin{array}{l}\text { Inpatient and outpatient services delivered in Danish } \\
\text { hospitals registered in the National Hospital } \\
\text { Register }\end{array}$ & $\begin{array}{l}\text { DRG tariffs and the DAGS were applied [28]. } \\
\text { Costs are understood as opportunity costs, i.e. } \\
\text { resources are scarce and resources spent in one area } \\
\text { cannot be spent in another [18]. Activities in the } \\
\text { Danish healthcare sector have been converted into a } \\
\text { monetary cost through the use of the DRG system. The } \\
\text { DRG tariffs are calculated from average operating } \\
\text { costs within each DRG group on a nationwide level } \\
\text { [28]. The system is developed for settlement of } \\
\text { accounts for patients in hospitals, individuals who } \\
\text { have chosen a hospital in another region than their } \\
\text { region of residence and patients in private hospitals. } \\
\text { This calculation produces approximate average costs, } \\
\text { which should be used with caution in economic } \\
\text { evaluations [29] }\end{array}$ \\
\hline $\begin{array}{l}\text { Primary care services delivered by GPs and privately } \\
\text { practising specialists } \\
\text { Visits by GPs, physiotherapists, chiropractors, } \\
\text { chiropodists, opticians and dietitians }\end{array}$ & $\begin{array}{l}\text { Reimbursement fees between the National Health } \\
\text { Insurance scheme and privately practising physicians } \\
\text { are used as cost units. GPs are compensated by regions } \\
\text { through a combination of a per capita fee } \\
\text { (approximately } 30 \% \text { of the total) and a fee-for-service } \\
\text { (approximately } 70 \% \text { ) [30]. To reflect this payment } \\
\text { scheme in the unit cost, } 43.8 \% \text { of the fee-for-service in } \\
\text { general practice was added to the cost. Overhead costs } \\
\text { covered by the capitation fee were distributed by } \\
\text { resource burden (and not across the number of visits) }\end{array}$ \\
\hline $\begin{array}{l}\text { Prescribed pharmaceuticals dispensed by Danish } \\
\text { pharmacies and registered in DNPrR. } \\
\text { (Pharmaceuticals consumed in hospitals are } \\
\text { included in DRG tariffs. Over-the-counter drugs are } \\
\text { not included in this analysis) }\end{array}$ & $\begin{array}{l}\text { Total sales price includes individuals' out-of-pocket } \\
\text { payments. Costs of prescribed pharmaceuticals are } \\
\text { shared between the individual and the primary } \\
\text { healthcare sector by a co-payment scheme in Denmark } \\
\text { in which individuals are reimbursed according to their } \\
\text { need. These costs were aggregated since total costs are } \\
\text { measured regardless of who pays. Although } \\
\text { pharmaceutical consumption includes VAT, Danish } \\
\text { healthcare services are exempted from VAT. As VAT is } \\
25 \% \text { in Denmark, } 20 \% \text { of pharmaceutical } \\
\text { consumption was subtracted to calculate comparable } \\
\text { net costs }\end{array}$ \\
\hline
\end{tabular}

DAGS, Danish Ambulatory Grouping System; DRG, diagnosis related grouping; DNPrR, Danish National Prescription Registry
Between 1 January 2001 and 31 December 2009, 139,075 people from our sampling frame were diagnosed with incident diabetes and included in the Danish National Diabetes Register. Of these, 13,992 (10\%) were in the screening group, and $125,083(90 \%)$ in the no-screening group. The groups were well balanced for age and citizenship (Table 2). There were slightly fewer men in the screening group (54\%) than the no-screening group (56\%). A larger proportion of the screening group had received over 15 years of education. Slightly lower proportions of the screening group had experienced IHD, stroke or cancer before the diagnosis of diabetes compared with the no-screening group.

Healthcare costs in the screening and no-screening groups Figures 2, 3, 4, 5 show the mean-adjusted annual costs per participant with incident diabetes by healthcare sector both before and after the time of diagnosis in the screening and no-screening groups. The results from the difference-in-difference regression analyses are presented in electronic supplementary material [ESM] Table 1.

After diagnosis, healthcare usage and subsequent costs were significantly higher in the no-screening group than the screening group. For example, primary care costs were approximately $5 \%$ higher in the no-screening group than the screening group during follow-up. The costs of redeemed medication were already higher in the no-screening group before diagnosis, but the difference between the groups increased further after diabetes diagnosis. Costs were approximately $12 \%$ higher in the no-screening group compared with the screening group in the 6 years following diagnosis. For 
Table 2 Characteristics of individuals with diabetes by screening group

\begin{tabular}{lll}
\hline Variable & $\begin{array}{l}\text { Screening group } \\
(N=13,992)\end{array}$ & $\begin{array}{l}\text { No-screening (control) } \\
\text { group }(N=125,083)\end{array}$ \\
\hline $\begin{array}{l}\text { Mean age at diagnosis } \\
\text { Male sex }\end{array}$ & $\begin{array}{l}59.9 \pm 7.7 \\
7495(53.6)\end{array}$ & $\begin{array}{l}59.2 \pm 9.2 \\
\text { Years of education }\end{array}$ \\
$\quad$ 0-10 & $5610(40.1)$ & $55,765(44.4)$ \\
$\quad 10-15$ & $6237(44.6)$ & $55,226(44.2)$ \\
$\quad>15$ & $2145(15.3)$ & $14,082(11.3)$ \\
European citizenship & $13,944(99.7)$ & $123,849(99)$ \\
Previous IHD $^{\mathrm{a}}$ & $1586(11.3)$ & $16,216(13)$ \\
Previous stroke $^{\mathrm{a}}$ & $628(4.5)$ & $6851(5.5)$ \\
Previous cancer $^{\mathrm{a}}$ & $2027(14.5)$ & $19,276(15.4)$ \\
\hline
\end{tabular}

Data are mean $\pm \mathrm{SD}$ or $n(\%)$

${ }^{a}$ Data taken from the National Patient Registry; data were included from 1994 until the date of diabetes diagnosis

inpatient and outpatient visits, there were no clear trends associated with the point of diabetes diagnosis. On average, however costs were lower among the screening group compared with the no-screening group both prior to and following diagnosis.

The mean annual cost estimates for primary care and redeemed medication were based on 2001-2012 data, while inpatient and outpatient costs were based on 2007-2012 data. The mean total cost difference is therefore estimated as the sum of the four mean annual cost components: inpatient and outpatient costs, primary care cost and cost of redeemed medication. After adjustment for confounders, annual healthcare costs per individual with incident diabetes were significantly lower in the screening group than the no-screening group across all healthcare sectors and for redeemed medication:

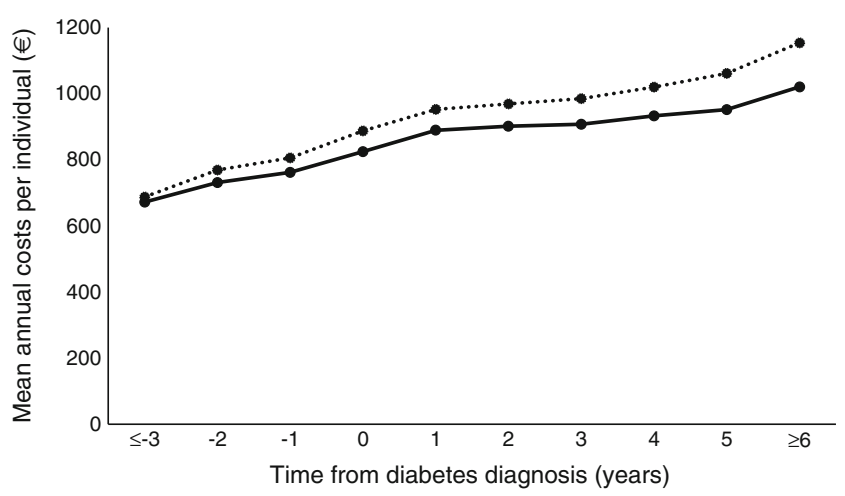

Fig. 2 Pharmaceutical costs per participant with incident diabetes 20012012 by intervention group according to time from diagnosis. Dotted line, costs for the no-screening (control) group; solid line, costs for the screening group. Black circles indicate that estimated mean differences in costs between the no-screening and screening groups in each time period are significantly different from zero $(p<0.05)$

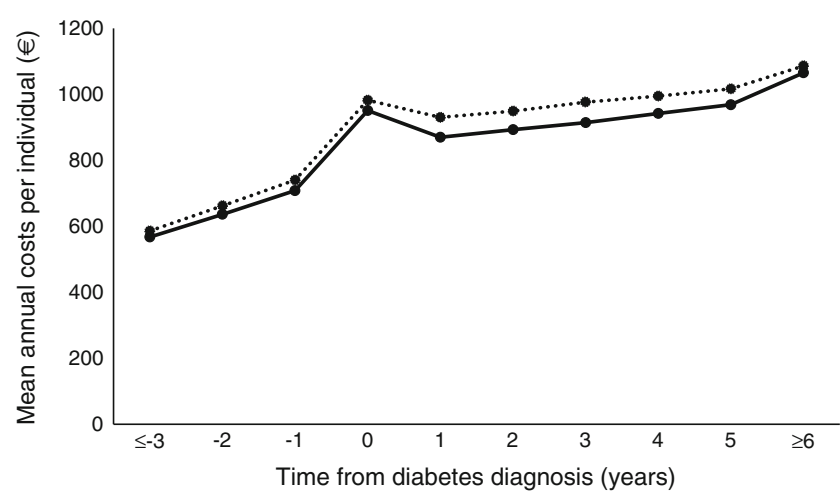

Fig. 3 Primary care costs per participant with incident diabetes 20012012 by intervention group according to time from diagnosis. Dotted line, costs for the no-screening (control) group; solid line, costs for the screening group. Black circles indicate that estimated mean differences in costs between the no-screening and screening groups in each time period are significantly different from zero $(p<0.05)$

annual difference in inpatient care costs $-€ 662(95 \% \mathrm{CI}$ $€ 865,-€ 459)$, outpatient care costs $€ 82$ (95\% CI $€ 157$, $-€ 7)$, primary care costs $€ 51,(95 \%$ CI $-€ 63,-€ 38)$ and pharmaceuticals $-€ 94$ (95\% CI $€ 111,-€ 76)$. The difference in mean total annual healthcare costs per individual with incident diabetes between groups was $€ 889$ (95\% CI $€ 1196$, $€ 581)$.

After subtracting the cost of introducing the ADDITION screening programme ( $€ 967$ per participant with incident diabetes) from the estimated annual healthcare cost savings ( $€ 889$ per participant with incident diabetes), and discounting this figure in the 5 years following the intervention, there was a cost saving associated with each individual with incident diabetes over a 5 year period of $€ 2688$ (95\% CI $€ 1421, € 3995$ ). This finding suggests that the intervention was associated with cost

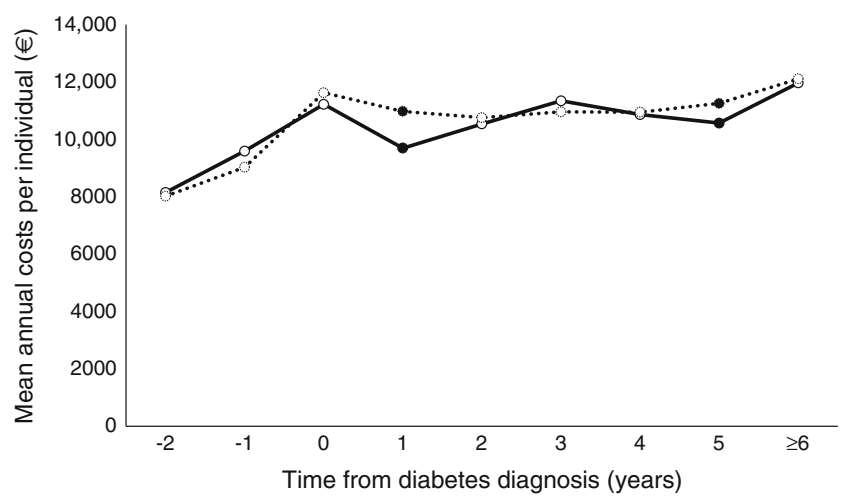

Fig. 4 Inpatient care costs per participant with incident diabetes $2007-$ 2012 by intervention group according to time from diagnosis. Dotted line, costs for the no-screening (control) group; solid line, costs for the screening group. Black circles, estimated mean differences in costs between the no-screening and screening groups in each time period are significantly different from zero $(p<0.05)$; white circles, there are no statistically significant estimated mean differences in costs in each time period $(p \geq 0.05)$ 


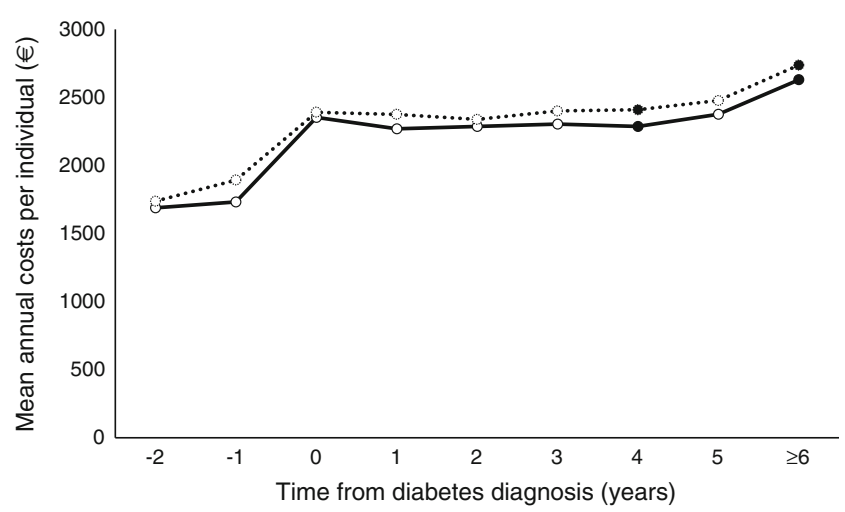

Fig. 5 Outpatient care costs per participant with incident diabetes $2007-$ 2012 by intervention group according to time from diagnosis. Dotted line, costs for the no-screening (control) group; solid line, costs for the screening group. Black circles, estimated mean differences in costs between the no-screening and screening groups in each time period are significantly different from zero $(p<0.05)$; white circles, there are no statistically significant estimated mean differences in costs in each time period $(p \geq 0.05)$

savings in the healthcare system within 2 years of the screening programme being introduced. Based on the 13,992 individuals with diabetes in the screening group, we estimate that the intervention saved the Danish healthcare system almost €37.6 million (95\% CI €19.8 million, $€ 55.3$ million) in the 5 years after the introduction of the screening programme.

Sensitivity analysis In our sensitivity analysis, the annual healthcare costs of the screening population assigned to routine care remained lower than costs in the no-screening group for: inpatient care $€ 633$ (95\% CI $€ 898,-€ 368$ ), primary care $€ 41$ (95\% CI -€58, € 24$)$ and medication -€90 (95\% CI $€ 117,-€ 63)$. The outpatient care costs were similar in both groups. As the costs of the screening programme within the routine care group were $€ 936$ per individual, and the total mean annual difference in costs between the screening and no-screening groups was $-€ 752$ (95\% CI $€ 1,169,-€ 335)$ per individual, ADDITION brings cost savings of $€ 2158$ (95\% CI $€ 444$; $€ 3871$ ) per individual within 5 years following the screening period. For the whole population of 13,992 individuals, the cost savings would be $€ 30.1$ million (95\% CI $€ 6.2$ million, €54.1 million).

\section{Discussion}

Our cost analysis of the ADDITION-Denmark screening programme showed that healthcare costs were significantly lower for individuals with incident type 2 diabetes in the screening group compared with the no-screening group. This was true for all examined health services - inpatient, outpatient and primary care-as well as for medication costs. We also showed that within 2 years of being introduced, ADDITION-Denmark was associated with cost savings in the healthcare system.

Our estimates of annual healthcare costs for individuals with diabetes in Denmark align well with the published literature. For example, a previous study [21] showed that the cost per individual with diabetes per year was $€ 6390$ in 2011 (compared with an annual mean of $€ 5910$ for the screening group and $€ 6854$ for the no-screening group between 2007 and 2012).

In ADDITION-Denmark, the relatively modest cost of screening per participant discovered to have diabetes was offset within 2 years by savings in the healthcare system. In terms of comparison, there are no other published trials that examine the costs of healthcare among individuals with incident type 2 diabetes in a screened group with those in an unscreened group. However, modelling studies show that screening for type 2 diabetes at the population level would be cost-effective over 30 and 50 years $[1,2,22]$.

Herman et al argue that that the benefits of screening and treatment primarily accrue from early diagnosis and by hastening the treatment of CVD risk factors during the lead time [3]. We have previously reported that ADDITION-Denmark brought forward the diagnosis of diabetes by 2.1 years and was associated with a significant reduction in mortality and CVD among those with diabetes [5]. We observed an increase in the proportion of screen-detected individuals who redeemed cardioprotective medication during follow-up [6]. However, larger proportions of clinically diagnosed individuals in the no-screening group redeemed medication compared with clinically diagnosed individuals in the screening group. As individuals in the no-screening group were diagnosed at a later stage in the disease trajectory, they may have had higher cholesterol, blood glucose and blood pressure values at diagnosis compared with the screening group, necessitating higher levels (and costs) of cardioprotective medication. The promotion of healthy behaviour change may also have impacted on CVD risk factors and CVD and mortality rates, and consequently lowered costs of healthcare among participants with diabetes in the screening group.

New drugs are more expensive, which might increase the costs based on these study data. However, new agents tend to be restricted to second- and third-line treatment, and hence may not have a major impact on our estimates of cost savings associated with screening in the first 510 years of the disease trajectory. In addition, preventive strategies for diabetes have gained traction in the past decade and may be associated with further cost savings. For example, the Diabetes Prevention Program in the USA [22] is being implemented through a number of different delivery channels [23, 24], and NHS England launched a National Diabetes Prevention Programme in 2016, which now covers 75\% of the English population 
$[25,26]$. Modelling studies suggest that these programmes will be cost-effective in the long term.

Strengths and limitations In this study, we used real-world, individual participant-level data to examine healthcare costs among participants with incident type 2 diabetes in a screened group compared with an unscreened group. We applied a healthcare sector perspective in our cost analysis. Only costs related to healthcare services and redeemed medication were included in the study. As such, costs relating to individuals' lost labour market productivity and utilisation of nursing services or other services such as transportation and helping aids in the house were not included in our analysis. These costs have previously been demonstrated to be high among people with diabetes, making up as much as $80 \%$ of the total societal costs [21, 23]. Furthermore, we did not include quality of life or the value of higher life expectancy in our analyses. Data on quality of life would enable a better comparison with the literature on the cost-utility of screening and prevention $[22$, 24-27].

We also did not include the healthcare cost savings accruing from an individual's death, as it will always be less expensive if a person dies and hence there is no argument for treatment or prevention [18].

A limitation of our study was the non-randomised design, as we cannot eliminate the possibility of selection bias and residual confounding. Groups were well balanced for the characteristics investigated at baseline. However, our findings might have been influenced by the higher levels of education and the slightly lower levels of pre-existing chronic disease in the intervention group. The cost of redeemed medication was also lower in the screening group before the introduction of the ADDITION-Denmark screening intervention. We adjusted for age, sex, education, GP county and prevalent chronic disease at diagnosis, which impacted on the size of the cost difference, although the final result remained significant. It is likely that adjusting for county took account of some of the potential socioeconomic differences across different regions in Denmark. We tried to minimize lead- and length-time biases by comparing costs for all individuals diagnosed with diabetes in the screening and no-screening groups.

We could have applied bootstrapping and cost acceptability curves to handle uncertainty [18]. As the intervention proved to be cost-saving within a short time period, we chose instead to use the lowest CI levels to describe uncertainty.

Conclusions Healthcare costs were lower among individuals with incident type 2 diabetes in a screened group compared with an unscreened group. The relatively modest cost of screening per participant discovered to have developed diabetes was offset within 2 years by savings in the healthcare system.
Acknowledgements We gratefully acknowledge the contribution of all participants, practice nurses and GPs in the ADDITION-Denmark study. Special thanks go to Marianne Pedersen (Department of Public Health, University of Aarhus) for her help in retrieving data from Statistics Denmark.

Data availability The data used in this paper was a combination of data obtained from the ADDITION study and data from national Danish registers at Statistics Denmark (DST). The ADDITION study data were obtained after a written application to the data owner, A. Sandbæk (Institute of Public Health, Aarhus University, Aarhus, Denmark; annelli.sandbaek@ph.au.dk). Data from DST were made available following an application to DST (www.dst.dk/en/TilSalg/ Forskningsservice). Data from ADDITION and DST were merged by DST and analyses were performed via a secure Virtual Private Network (VPN) connection. In accordance with the Danish Act on Processing of Personal Data, future interested researchers must perform the steps mentioned above to obtain access to the data.

Funding The Danish Diabetes Association (Diabetesforeningen) provided funds to complete the work on this paper. ADDITION-Denmark was supported by the National Health Services in the counties of Copenhagen, Aarhus, Ringkøbing, Ribe and South Jutland in Denmark, the Danish Council for Strategic Research, the Danish Research Foundation for General Practice, Novo Nordisk Foundation, the Danish Centre for Evaluation and Health Technology Assessment, the diabetes fund of the National Board of Health, the Danish Medical Research Council and the Aarhus University Research Foundation. The trial has been supported by unrestricted grants from Novo Nordisk AS, Novo Nordisk Scandinavia AB, Novo Nordisk UK, ASTRA Denmark, Pfizer Denmark, GlaxoSmithKline Pharma Denmark, Servier Denmark AS and HemoCue Denmark AS. SJG's research programme is supported by the MRC Epidemiology Unit core funding (MC_UU_12015/4).

Duality of interest Applied Economics and Health Research received a grant from the Danish Diabetes Association to conduct the analysis. The Danish Diabetes Association has not had any access to data, analyses or conclusions from this paper. AS reports receiving lecture fees for providing continuing medical education to GPs. SJG is a National Institute of Health Research (NIHR) Senior Investigator and member of the NIHR School for Primary Care Research. SJG receives an honorarium and reimbursement of travel expenses from Eli Lilly associated with membership of an independent data-monitoring committee for a randomised trial of a medication to lower glucose levels. SJG also received an honorarium from Janssen for speaking at an educational meeting in 2015. TL holds shares in Novo Nordisk A/S. TL reports receiving fees for lecturing for Danish GPs and attending two international board meetings for Astra Zeneca on early detection and treatment of diabetes within the last 2 years. RKS is supported by the Health Foundation's grant to the University of Cambridge for The Healthcare Improvement Studies (THIS) Institute. RKS was previously supported by the Aarhus Institute of Advanced Studies and the Danish Diabetes Academy under a visiting professorship to complete part of this work. The Danish Diabetes Academy is funded by the Novo Nordisk Foundation.

Contribution statement CS and RKS had full access to all of the data in the study and take responsibility for the integrity of the data and the accuracy of the data analysis. CS conducted and is responsible for the data analysis. AS and TL designed the ADDITIONDenmark study together with Knut Borch-Johnsen, and are principal investigators for the trial. CS, RKS and TL conceived the study question for this paper and developed the study proposal. CS and RKS participated in acquisition of the data from Statistics Denmark. All authors participated in interpretation of the data and critical revision of the report for important intellectual content. CS, AK, TL and RKS provided administrative, technical and material support 
for the study. All authors approved the final version of the paper to be published. TL acts as guarantor for this paper.

Open Access This article is distributed under the terms of the Creative Commons Attribution 4.0 International License (http://creativecommons. org/licenses/by/4.0/), which permits unrestricted use, distribution, and reproduction in any medium, provided you give appropriate credit to the original author(s) and the source, provide a link to the Creative Commons license, and indicate if changes were made.

\section{References}

1. Kahn R, Alperin P, Eddy D et al (2010) Age at initiation and frequency of screening to detect type 2 diabetes: a cost-effectiveness analysis. Lancet 375:1365-1374

2. Schuetz CA, Alperin P, Guda S et al (2013) A standardized vascular disease health check in europe: a cost-effectiveness analysis. PLoS One 8:e66454

3. Herman WH, Ye W, Griffin SJ et al (2015) Early detection and treatment of type 2 diabetes reduce cardiovascular morbidity and mortality: a simulation of the results of the Anglo-Danish-Dutch Study of Intensive Treatment in People With Screen-Detected Diabetes in Primary Care (ADDITION-Europe). Diabetes Care 38:1449-1455

4. Simmons RK, Echouffo-Tcheugui JB, Sharp SJ et al (2012) Screening for type 2 diabetes and population mortality over 10 years (ADDITION-Cambridge): a cluster-randomised controlled trial. Lancet 380:1741-1748

5. Simmons RK, Griffin SJ, Witte DR, Borch-Johnsen K, Lauritzen T, Sandbaek A (2017) Effect of population screening for type 2 diabetes and cardiovascular risk factors on mortality rate and cardiovascular events: a controlled trial among 1,912,392 Danish adults. Diabetologia 60:2183-2191

6. Simmons RK, Griffin SJ, Lauritzen T, Sandbaek A (2017) Effect of screening for type 2 diabetes on risk of cardiovascular disease and mortality: a controlled trial among 139,075 individuals diagnosed with diabetes in Denmark between 2001 and 2009. Diabetologia 60:2192-2199

7. Lauritzen T, Griffin S, Borch-Johnsen K et al (2000) The ADDITION study: proposed trial of the cost-effectiveness of an intensive multifactorial intervention on morbidity and mortality among people with type 2 diabetes detected by screening. Int $\mathbf{J}$ Obes Relat Metab Disord J Int Assoc Study Obes 24(Suppl 3): S6-S11

8. Christensen JO, Sandbaek A, Lauritzen T, Borch-Johnsen K (2004) Population-based stepwise screening for unrecognised type 2 diabetes is ineffective in general practice despite reliable algorithms. Diabetologia 47:1566-1573

9. Griffin SJ, Borch-Johnsen K, Davies MJ et al (2011) Effect of early intensive multifactorial therapy on 5-year cardiovascular outcomes in individuals with type 2 diabetes detected by screening (ADDITION-Europe): a cluster-randomised trial. Lancet 378: 156-167

10. Glumer C, Carstensen B, Sandbaek A, Lauritzen T, Jorgensen T, Borch-Johnsen K (2004) A Danish diabetes risk score for targeted screening: the Inter99 study. Diabetes Care 27:727-733
11. Conroy RM, Pyorala K, Fitzgerald AP et al (2003) Estimation of ten-year risk of fatal cardiovascular disease in Europe: the SCORE project. Eur Heart J 24:987-1003

12. Alberti KG, Zimmet PZ (1998) Definition, diagnosis and classification of diabetes mellitus and its complications. Part 1: diagnosis and classification of diabetes mellitus provisional report of a WHO consultation. Diabet Med J Br Diabet Assoc 15:539-553

13. Carstensen B, Kristensen J, Ottosen P, Borch-Johnsen K (2008) The Danish National Diabetes Register: trends in incidence, prevalence and mortality. Diabetologia 51:2187-2196

14. Green A, Sortso C, Jensen PB, Emneus M (2015) Validation of the Danish National Diabetes Register. Clin Epidemiol 7:5-15

15. UNESCO (1997) International Standard Classification of Education 1997. United National Educational, Scientific and Cultural Organization, Paris

16. Thygesen LC, Daasnes C, Thaulow I, Hansen HB (2011) Introduction to Danish (nationwide) registers on health and social issues: structure, access, legislation and archiving. Scand J Public Health 39(Suppl 7):12-16

17. Dalsgaard EM, Christensen JO, Skriver MV, Borch-Johnsen K, Lauritzen T, Sandbaek A (2010) Comparison of different stepwise screening strategies for type 2 diabetes: finding from Danish general practice, ADDITION-DK. Prim Care Diabetes 4:223-229

18. Drummond M, Sculper M, Torrance G, O'Brien B, Stoddart G (2005) Methods for the economic evaluation of health care programmes. Oxford University Press, New York

19. Ettaro L, Songer TJ, Zhang P, Engelgau MM (2004) Cost-of-illness studies in diabetes mellitus. PharmacoEconomics 22:149-164

20. Bech M, Gyrd-Hansen D (2005) Effects coding in discrete choice experiments. Health Econ 14:1079-1083

21. Sortsø C, Green A, Jensen P, Emneus M (2015) Societal costs of diabetes mellitus in Denmark. Diabet Med 33:877-885

22. Gillies CL, Lambert PC, Abrams KR et al (2008) Different strategies for screening and prevention of type 2 diabetes in adults: cost effectiveness analysis. BMJ 336:1180-1185

23. American Diabetes Association (2013) Economic costs of diabetes in the U.S. in 2012. Diabetes Care 36:1033-1046

24. Zhong Y, Lin PJ, Cohen JT, Winn AN, Neumann PJ (2015) Costutility analyses in diabetes: a systematic review and implications from real-world evidence. Value Health 18:308-314

25. van Giessen A, Boonman-de Winter LJ, Rutten FH et al (2016) Cost-effectiveness of screening strategies to detect heart failure in patients with type 2 diabetes. Cardiovasc Diabetol 15:48

26. Corey KE, Klebanoff MJ, Tramontano AC, Chung RT, Hur C (2016) Screening for nonalcoholic steatohepatitis in individuals with type 2 diabetes: a cost-effectiveness analysis. Dig Dis Sci 61:2108-2117

27. Neidell M, Lamster IB, Shearer B (2017) Cost-effectiveness of diabetes screening initiated through a dental visit. Community Dent Oral Epidemiol 45:275-280

28. Ministeriet-sundhedogforebyggelse (2012) DRG-leksikon. Available from http://www.sum.dk/Sundhed/DRG-systemet/ DRG-leksikon.aspx. Accessed 30 April 2012

29. Pedersen KM (2010) [DRG again again]. Ugeskr Laeger 172:2205 [article in Danish]

30. Kristensen T, Rose Olsen K, Sortsø C, Ejersted C, Thomsen JL, Halling A (2013) Resources allocation and health care needs in diabetes care in Danish GP clinics. Health Policy 113:206-215 